\title{
BMJ Open Primary care interventions to reduce cardiovascular risk behaviours in adolescents: a protocol for a systematic review
}

\author{
Dagmar M Haller, ${ }^{1,2,3}$ Eva Pfarrwaller, ${ }^{1,4}$ Bernard Cerutti, ${ }^{5}$ Jean-Michel Gaspoz ${ }^{6,7}$
}

To cite: Haller DM,

Pfarrwaller E, Cerutti B, et al. Primary care interventions to reduce cardiovascular risk behaviours in adolescents: a protocol for a systematic review. BMJ Open 2016;6 e011936. doi:10.1136/ bmjopen-2016-011936

- Prepublication history and additional material is available. To view please visit the journal (http://dx.doi.org/ 10.1136/bmjopen-2016011936).

Received 16 March 2016 Revised 28 July 2016 Accepted 3 August 2016

CrossMark

For numbered affiliations see end of article.

Correspondence to Dr Dagmar M Haller; dagmar. haller-hester@hcuge.ch

\section{ABSTRACT}

Introduction: Health-compromising behaviours are often acquired in adolescence. Alongside broader public health interventions, preventive interventions within primary care have the potential to encourage long-lasting behaviour change by tailoring messages to each individual. The aim of this study is to determine the effectiveness of primary care interventions in reducing the 3 main cardiovascular risk behaviours (smoking, low physical activity and unhealthy diet) in adolescents aged 10-19 years. It is also to identify successful initiatives and ingredients for such success that could be replicated in primary care.

Methods and analysis: This systematic review of the literature and meta-analysis will follow the Preferred Reporting Items for Systematic Reviews and MetaAnalyses (PRISMA) recommendations. The following databases will be searched for articles published between January 1990 and December 2016: MEDLINE, EMBASE, PsychINFO, CINAHL, Cochrane Central Register of Controlled Trials, ClinicalTrials.gov, ISRCTN registry. Our search will focus on randomised and cluster randomised controlled trials of interventions conducted in primary care practices to reduce the 3 main cardiovascular risk behaviours in adolescents aged 10-19 years, compared with active (information leaflet, etc) or passive (usual care, etc) control conditions. The primary outcomes will be smoking, physical activity and diet, measured either objectively or by self-report. Secondary outcomes such as body mass index or insulin resistance will also be examined. 2 reviewers will independently screen articles, extract relevant data and assess study quality using the Cochrane risk of bias tool. A meta-analysis will be considered if the number of studies is sufficient and outcomes are sufficiently homogeneous. The Grading of Recommendations Assessment, Development and Evaluation (GRADE) criteria will be used to assess the quality of the evidence. Ethics and dissemination: This systematic review will add to our knowledge on the prevention of cardiovascular disease early in life and these findings will be disseminated through peer-reviewed publications and presentations at relevant conferences. Study registration number: PROSPERO CRD42016028045.

\section{Strengths and limitations of this study}

- This review will use a systematic and comprehensive approach to add to our knowledge about early prevention of cardiovascular disease in primary care.

- It will help identify successful initiatives as well as ingredients for such success in order to contribute to reducing cardiovascular risk in adolescence.

- Since this is a neglected area of prevention research, the number of high-quality studies included in the review is likely to be limited.

- A high level of heterogeneity both between interventions and outcome measurements could limit the strength of the findings emerging from this review.

\section{INTRODUCTION}

In $2015,25 \%$ of all deaths in the world are to be caused by cardiovascular disorders. ${ }^{1}$ These disorders represent the first cause of adult mortality and morbidity in high-income countries and are a growing concern in lowincome and middle-income countries as well. $^{23}$

Health-compromising behaviours acquired in adolescence and young adulthood are major contributors to the development of cardiovascular disorders in later life. ${ }^{4}$ Thus, adolescence represents both a period of risk for the acquisition of such behaviours and a period of opportunity to prevent them. Smoking, low physical activity and unhealthy diet are the most prominent cardiovascular risk behaviours in adolescence. ${ }^{4} 5$ Findings from population-based surveys such as the HBSC-2010 survey (Health Behaviours in School-Aged Children, in more than 40 highincome and middle-income countries) show that a significant proportion of adolescents are exposed to these risk factors: at the age of $15,20 \%$ smoke at least once a week 
(up from $1 \%$ at the age of 11 years), two-thirds of adolescents have sedentary behaviours (watching television 2 or more hours a day on weekdays), more than $80 \%$ of them report $<1$ hour a week of moderate-to-vigorous exercise, less than a third eat fruit daily, and $10 \%$ of girls and $20 \%$ of boys are overweight or obese. ${ }^{6}$

Population approaches may be successful to improve diet, physical activity and smoking habits. ${ }^{7}$ Individual approaches at the healthcare level are also important, however, to individualise public health messages and encourage behaviour change. ${ }^{7}$ Within a life-course approach to adolescent health, primary care physicians (PCPs) are ideally placed to promote healthy lifestyles and offer preventive care that may influence young people's health well into adulthood. ${ }^{8}$ PCPs' role has been highlighted both in the WHO's report on noncommunicable diseases and in national strategies. ${ }^{9} 10$ Despite increased recognition of the added value of preventive care, evidence is needed to support its provision since preventive care is time-consuming and often financially unrewarding for practitioners. ${ }^{11}$ Until now, little focus has been placed on the effectiveness of preventive interventions in childhood to ensure ideal cardiovascular health and reduce cardiovascular risk later in life. ${ }^{12}$ We found no previous review of the evidence in relation to interventions for the prevention of the three main cardiovascular risk behaviours in adolescents attending primary care.

The aim of this systematic review of the literature is therefore to determine the effectiveness of primary care interventions in reducing the three main cardiovascular risk behaviours in adolescents. Our objectives are to:

1. Identify and summarise the evidence about the effectiveness of interventions in primary care to reduce the three main cardiovascular risk behaviours (smoking, low physical activity and unhealthy diet) in adolescents (aged 10-19 years, according to the WHO definition).

2. Identify the features associated with the effectiveness of interventions, such as communication style (eg, the use of motivational interviewing), mode of delivery (eg, entirely face to face or supported by a webbased tool), duration and frequency.

3 . If possible, to measure the effect size of the interventions in reducing each of the three main cardiovascular risk behaviours.

\section{METHODS AND ANALYSIS}

This protocol follows the Preferred Reporting Items for Systematic Reviews and Meta-Analyses Protocols (PRISMA-P) guidelines. ${ }^{13}$ It is registered in the PROSPERO registry (CRD42016028045).

\section{Eligibility criteria}

Study characteristics

Inclusion will be limited to randomised controlled trials (RCTs) and cluster RCTs (cRCTs) published in any language (but with an abstract in English) between January 1990 and December 2015. Completed but yet unpublished trials identified through trial registration platforms will also be eligible for inclusion. Non-RCTs, controlled before-after studies and interrupted time series will, however, also be considered if the number of identified RCTs is low (five or less).

\section{Population}

Studies will be considered if the tested intervention targets adolescents between the ages of 10 and 19 years attending a primary care practice. Studies including participants younger than 10 years will also be included if data in relation to the target age group can be analysed separately. Since young adults are often considered within a developmental continuum from adolescence into adulthood, studies involving participants up to the age of 24 years will also be eligible, as long as they also include adolescents aged between 10 and 19 years and provide data for this age group that can be considered separately.

\section{Intervention}

Interventions that aim to address the three main cardiovascular risk behaviours, either alone or in combination, will be eligible. The aim of the interventions should be to reduce tobacco use, increase physical activity/reduce sedentary behaviour and/or promote a healthy diet (fulfilling criteria for a healthy diet of the American Heart Association). ${ }^{14}$ Interventions delivered by the PCP or any other professional working within the primary care practice will be considered, as well as interventions involving multimedia tools targeting patients identified in the primary care practice.

\section{Comparator or control}

Studies comparing the intervention either to an active control (eg, information leaflet, intervention by specialist) or passive control (eg, usual care, waiting list, etc) will be eligible.

\section{Outcomes}

Studies will be included if they assess the effect of the intervention on one or several of the following outcomes:

- Smoking: objectively measured (cotinine levels) and/ or self-reported (subjective);

- Physical activity/sedentary behaviour assessed either objectively (pedometer, etc) or subjectively, preferably using standardised tools;

- Diet: objectively measured (biomarkers for dietary habits) and/or self-reported (subjective), preferably using standardised tools.

Secondary outcomes: for studies of preventive interventions targeting physical activity and/or dietary habits, outcomes such as body mass index (BMI), blood pressure, blood insulin levels will also be recorded if available. 


\section{Data sources and search}

The following databases will be searched for articles published between January 1990 and December 2016: MEDLINE, EMBASE, PsychINFO, CINAHL, Cochrane Central Register of Controlled Trials, ClinicalTrials.gov, ISRCTN registry. We will also search the reference lists of included studies and contact authors of included studies to ask about their knowledge of any additional studies not identified in the initial search.

The search strategy will use a combination of medical subject headings (MeSH) and free-text words for the population, intervention, outcomes and study design, adapted to each database. No limits will be applied. Table 1 presents the initial keywords on which the full search strategy will be developed. A search strategy for PubMed is presented in online supplementary appendix 1 .

\section{Data management}

Endnote software will be used for data management. All the results of literature searches will be imported into the program and duplicates removed by the main reviewer.

\section{Selection process}

Selection of relevant studies will follow the PRISMA guidelines, using a three-step process, all carried out by two reviewers, with a third reviewer available to help resolve any disagreement. All identified titles and abstracts will first be screened for inclusion/exclusion criteria, and then the full texts of potentially eligible articles meeting inclusion criteria on the basis of their title/ abstract. At each step of the selection process, reasons for inclusion/exclusion will be recorded.

\section{Data extraction}

Using a process similar to the one developed in a previous systematic review by our team, we will develop and

\begin{tabular}{ll}
$\begin{array}{l}\text { Table } 1 \\
\text { will be built }\end{array}$ & \\
\hline PICOS & Keywords \\
\hline Population & $\begin{array}{l}\text { Adolescents, Teenagers, Youth } \\
\text { Primary Health Care, Family practice, } \\
\text { General practice }\end{array}$ \\
& $\begin{array}{l}\text { General practitioner, Family practitioner, } \\
\text { Family doctor, Family physician, Primary } \\
\text { Care Physician }\end{array}$ \\
Intervention & $\begin{array}{l}\text { Intervention, brief intervention, behavior } \\
\text { change, counselling }\end{array}$ \\
Comparator/ & $\begin{array}{l}\text { NA (no specific control condition will be } \\
\text { searched in order to avoid narrowing the } \\
\text { control }\end{array}$ \\
Search) \\
Outcome & $\begin{array}{l}\text { Smoking, Physical activity, Diet, } \\
\text { cardiovascular risk }\end{array}$ \\
Study design & $\begin{array}{l}\text { Randomized controlled trial, cluster } \\
\text { randomized controlled trial }\end{array}$ \\
\hline
\end{tabular}

pilot test a data extraction form to record the following data: study authors, country, setting, population and participant sociodemographic characteristics, interventions and their specific features, control condition, outcomes (both subjective and objective, at all follow-up time points) and timing of measurements, and information to be used to assess risk of bias. ${ }^{15}$ Two reviewers will independently test the data extraction form on three articles and the form will then be adapted to ensure the reliability of the data extraction process. The two reviewers will then extract data for each included study independently and any disagreement will be resolved through discussion, if needed involving the third reviewer in order to reach consensus.

\section{Missing data}

Attempts will be made to contact study authors via email to obtain clarification if data are incomplete in the study report and we will allow a delay of 6 weeks to receive a response following two email attempts. We do not plan to use imputation to deal with missing data.

\section{Assessing risk of bias}

Two reviewers will independently assess risk of bias for each included study. They will use the tool developed by the Cochrane Collaboration to assess and report risk of bias in the following domains: sequence generation, allocation concealment, blinding of participants and researchers, blinding of outcome assessors, incomplete outcome data, selective outcome reporting, and other sources of bias (including conflicts of interest and study sponsorship). ${ }^{16}$ Risk of bias will be described as low, unclear or high, and reasons for each assessment will be documented.

\section{Evidence synthesis}

We will produce a narrative synthesis of the data extracted from the included studies and present it in a text and summary tables. In addition to the evidence in relation to the effectiveness of different interventions, a special emphasis will be placed on presenting key components of successful interventions.

\section{Data analysis}

Descriptive statistics will be used to summarise general data in particular in relation to participants and outcomes. If the number and homogeneity of retrieved studies are sufficient, we will pool outcome estimates of intervention effectiveness and conduct meta-analyses using random-effects models. Separate meta-analyses will be conducted for each of the three risk behaviour outcomes. If multiple measures are used to measure an outcome, we will favour meta-analysis of subjective measures as they are likely to be reported in the largest number of studies. However, we also plan to conduct a meta-analysis of outcomes measured objectively if these are reported in more than two studies. Long-term outcomes will be favoured over short-term outcomes for the 
meta-analysis if the number of studies reporting them is sufficient. If length of follow-up is very variable, these data will be introduced as co-variates in the analyses.

Dichotomous outcomes will be presented as risk ratios with $95 \%$ CIs. For continuous outcomes, effect sizes will be presented using either weighted mean differences when similar outcome measures have been used or, if possible, standardised mean differences where different measures of a same outcome have been used.

Statistical heterogeneity will be explored using the $\mathrm{I}^{2}$ statistic. If substantial heterogeneity $\left(\mathrm{I}^{2}>50 \%\right)$ is present and the number of included studies is sufficient, we will use subgroup analyses (eg, younger vs older adolescent participants, interventions targeting one vs several risk behaviours, single session vs multiple component intervention, etc) to attempt to identify reasons for heterogeneity. If some studies are at high risk of bias, sensitivity analyses will be conducted excluding these studies to assess the extent to which they influence the findings.

\section{Assessment of possible reporting bias}

If the number of studies included in a meta-analysis is sufficient ( $\sim 10$ studies), we will investigate possible reporting bias using funnel plots.

\section{Assessment of the strength of the evidence}

The quality of the evidence in relation to the effect of interventions on the three main outcomes will be assessed using the Grading of Recommendations Assessment, Development and Evaluation (GRADE) guidelines. ${ }^{17}$ The evidence will be assessed across the following domains: design and risk of bias, consistency, directness, precision and publication bias (which includes possible role of sponsor)

\section{ETHICS AND DISSEMINATION}

Ethics approval will not be necessary as no primary patient data will be collected.

Worldwide, the increasing burden of disease due to non-communicable diseases, including cardiovascular diseases, has shed light on the need to place a stronger emphasis on prevention. Interventions targeting adolescents can act at an age when risk behaviours spread their roots and have the potential to influence the health of individuals throughout their adult life. Alongside broader public health interventions, preventive interventions within primary care have the potential to encourage long-lasting behaviour change by better tailoring messages to each individual. This systematic review will add to our knowledge on the prevention of cardiovascular disease. In particular, it will help us identify evidence-based primary care interventions for adolescents for which implementation efforts should be promoted. It will allow us to identify successful initiatives and, hopefully, also ingredients for such success, as well as possible gaps in knowledge in relation to effective interventions opening paths for further research. These findings will be disseminated through peer-reviewed publications and presentations in relevant conferences. Our review will also serve to define a future research agenda in this as yet much neglected domain of prevention research.

Author affiliations

${ }^{1}$ Primary Care Unit, Faculty of Medicine, University of Geneva, Geneva, Switzerland

${ }^{2}$ Adolescent and Young Adult Program, Department of Community, Primary Care and Emergency Medicine and Department of Pediatrics, Geneva

University Hospitals, Geneva, Switzerland

${ }^{3}$ Department of General Practice, The University of Melbourne, Melbourne, Australia

${ }^{4}$ Geneva School Health Service, Geneva, Switzerland

${ }^{5}$ Faculty of Medicine, UDREM, University of Geneva, Geneva, Switzerland

${ }^{6}$ Faculty of Medicine, Institute of Primary Care, University of Geneva, Geneva,

Switzerland

${ }^{7}$ Division of Primary Care, Department of Community, Primary Care and Emergency Medicine, Geneva University Hospitals, Geneva, Switzerland

Acknowledgements This protocol was informed by previous work conducted for her Master in Medicine thesis by Marion Walker, under the supervision of $\mathrm{DMH}$.

Contributors DMH conceptualised the review and drafted the manuscript. She is the guarantor. EP, BC and J-MG critically revised the protocol. EP developed the search strategy presented in the protocol, together with $\mathrm{DMH}$. All authors approved the final version of the protocol for publication.

Funding This systematic review will be funded by a grant from the Gottfried und Julia Bangerter-Rhyner Foundation for health services research (Swiss Academy of Medical Sciences).

Competing interests None declared.

Provenance and peer review Not commissioned; externally peer reviewed.

Open Access This is an Open Access article distributed in accordance with the Creative Commons Attribution Non Commercial (CC BY-NC 4.0) license, which permits others to distribute, remix, adapt, build upon this work noncommercially, and license their derivative works on different terms, provided the original work is properly cited and the use is non-commercial. See: http:// creativecommons.org/licenses/by-nc/4.0/

\section{REFERENCES}

1. World Health Organization. Projections of mortality and causes of death, 2015 and 2030. Geneva: World Health Organization, 2014.

2. Lozano R, Naghavi M, Foreman K, et al. Global and regional mortality from 235 causes of death for 20 age groups in 1990 and 2010: a systematic analysis for the Global Burden of Disease Study 2010. Lancet 2012;380:2095-128.

3. Murray CJ, Vos T, Lozano R, et al. Disability-adjusted life years (DALYs) for 291 diseases and injuries in 21 regions, 1990-2010: a systematic analysis for the Global Burden of Disease Study 2010. Lancet 2012;380:2197-223.

4. Laitinen TT, Pahkala K, Magnussen CG, et al. Ideal cardiovascular health in childhood and cardiometabolic outcomes in adulthood: the Cardiovascular Risk in Young Finns Study. Circulation 2012;125:1971-8.

5. Haas GM, Bertsch T, Schwandt P. Prehypertension and cardiovascular risk factors in children and adolescents participating in the community-based prevention education program family heart study. Int J Prev Med 2014;5(Suppl 1):S50-6.

6. Currie C, Zanotti C, Morgan A, et al. Social determinants of health and well-being among young people. Health Behaviour in School-aged Children (HBSC) study: international report from the 2009/2010 survey. (Health Policy for Children and Adolescents, No. 6). Copenhagen: WHO Regional Office for Europe, 2012.

7. Mozaffarian D, Afshin A, Benowitz NL, et al. Population approaches to improve diet, physical activity, and smoking habits: a scientific statement from the American Heart Association. Circulation 2012;126:1514-63. 
8. Sawyer SM, Afifi RA, Bearinger LH, et al. Adolescence: a foundation for future health. Lancet 2012;379:1630-40.

9. World Health Organization. Global status report on noncommunicable diseases 2014. Geneva: WHO, 2014

10. Office fédéral de la santé publique (OFSP), Conférence suisse des directrices et directeurs cantonaux de la santé (CDS). Stratégie nationale de prévention des maladies non transmissibles (stratégie MNT): projet. Berne: OFSP, 2015.

11. Yarnall KS, Pollak KI, Ostbye T, et al. Primary care: is there enough time for prevention? Am J Public Health 2003;93:635-41.

12. Hayman LL, Meininger JC, Daniels SR, et al. Primary prevention of cardiovascular disease in nursing practice: focus on children and youth: a scientific statement from the American Heart Association Committee on Atherosclerosis, Hypertension, and Obesity in Youth of the Council on Cardiovascular Disease in the Young, Council on Cardiovascular Nursing, Council on Epidemiology and Prevention, and Council on Nutrition, Physical Activity, and Metabolism. Circulation 2007;116:344-57.

13. Shamseer L, Moher D, Clarke M, et al. Preferred reporting items for systematic review and meta-analysis protocols (PRISMA-P) 2015: elaboration and explanation. BMJ 2015;349:g7647.

14. Gidding SS, Dennison BA, Birch LL, et al. Dietary recommendations for children and adolescents: a guide for practitioners: consensus statement from the American Heart Association. Circulation 2005;112:2061-75.

15. Pfarrwaller E, Sommer J, Chung C, et al. Impact of interventions to increase the proportion of medical students choosing a primary care career: a systematic review. J Gen Intern Med 2015;30:1349-58.

16. Higgins J, Green S, eds. Cochrane handbook for systematic reviews of interventions. The Cochrane Collaboration, 2011.

17. Guyatt G, Oxman AD, Sultan $\mathrm{S}$, et al. GRADE guidelines: 11, making an overall rating of confidence in effect estimates for a single outcome and for all outcomes. J Clin Epidemiol 2013;66:151-7. 